\title{
Acknowledgment of Ad Hoc Reviewers (2017)
}

The editors acknowledge the generous and valuable help from the following reviewers who, in addition to the Editorial Board members, reviewed manuscripts submitted for publication on an ad hoc basis for the 2017 volume.

\section{Becci Akin}

Deidre M. Anglin

Laura Armstrong

Mary I. Armstrong

Thalida Arpawong

Shervin Assari

Shalhevet Attar-Schwartz

Majdouline Aziz

Christina Balderrama-Durbin

Martha E. Banks

Armando Barragán

Michelle Greenspoon Barrett

Joy Baumgartner

John Belcher

Kia Bentley

Dolores Bigfoot

Gary Blau

Arthur Blume

Jessica Borelli

Wendy Bostwick

Axel Bouchard

Orna Braun-Lewensohn

Britney Brinkman

Aerika Brittian Loyd

Elizabeth Bromley

Catana Brown

Tony Brown

Eric Bruns

Ann Buchanan

Amy C. Butler

Deborah Bybee

Will H. Canu

Giuseppe Carrà

Tony Cassidy

Gabriel Cesar

Fang-Pei Chen

Kee Hong Choi

Krista Chronister

Ann Coker

Jennifer Cole

Cyleste C. Collins

Nadine Connell

Anne Conway

Amy Cook

Shauna Cooper

Mark E. Courtney

Shantel Crosby

E. Mark Cummings

Mireille Cyr

Danielle Dallaire

Fran Danis

Graham Danzer

Nihaya Daoud

Larry Davidson

Carolyn Joy Dayton

Scott Decker

Rachel Dekel

Hector Del Castillo

James Densley

Melissa Dichter

Julia Dickson-Gomez
Norin Dollard

David Matthew Doyle

Jeffrey Draine

Christopher F. Drescher

Kristin Duppong-Hurley

Amy Dworsky

M. Ann Easterbrooks

Nicholas Eaton

Katie Edwards

Christopher Ellison

Christopher Erbes

Mary E. Evans

Caroline Evans

Erika Felix

Mark Ferro

Matia Finn-Stevenson

Kevin M. Fitzpatrick

Johanna B. Folk

Julian Ford

Larry Gant

Elizabeth Gassin

Noni K. Gaylord-Harden

Deborah Gioia

Jessica R. Goodkind

Sara Goodkind

Lisa Goodman

Brenna Greenfield

Robert Griffore

Andrew Grogan-Kaylor

Alan Gross

Meekyung Han

Annie Harper

William English Hartmann

Robin Harwood

Kirsten Havig

Claire Henderson

Susan Henney

Mario Hernandez

Craig Higson-Smith

Devon Hinton

Stevan Hobfoll

Steven Hoffman

Jun Sung Hong

Cindy Huang

Jonathan C. Huefner

Jaclyn White Hughto

Sarah Hurley

Ephrat Huss

Nicholas Ialongo

Jay A. Irwin

Anthony Jorm

Femmie Juffer

Samuel Juni

Zornitsa Kalibatseva

Hugo Kamya

Jonathan Kanter
Carmit Katz

Traci M. Kennedy

Angie Kennedy

Vivian Khamis

Siamak Khodarahimi

Mona Khoury-Kassabri

Sean Kidd

Mimi Kim

Maggie Kirkman

Rolf J. Kleber

Erik J. Knorth

Sharon F. Lambert

Osnat Lavenda

Iris Lavi

Justin Lavner

Bethany R. Lee

Kirsty Lee

Malena Lenta

Amber Letcher

Terri Lewis

Junlei Li

Susan Limber

Maya Lis-Turlejska

Alicia Lucksted

Paul Lysaker

Carolina Marin

Nathan Martin

Alexa Martin-Storey

Bryan McCormick

Lucy Sarah McGregor

Barbara McMorris

Amy Mendenhall

Paul Miller

Kimberly Mitchell

Eduardo S. Morales

Melissa Lynne Morgan Consoli

Kathleen Moritz Rudasill

Linda James Myers

Yochay Nadan

Julie L. Nagoshi

Angela Narayan

Timothy D. Nelson

La Tonya Noel

Peter Norton

Jennifer O'Brien

Kurt Organista

Philip Osteen

Joan Ostrove

Aasim Padela

Heather Pane Seifert

Yin Paradies

Einat Peled

Dan Perlman

Brandy Pina-Watson

Ricardo José Pinto

Shirley Porterfield

V. Paul Poteat
Seth Prins

David Pyrooz

Yvonne Rafferty

Giora Rahav

Melisa Rempfer

Harmony R. Rhoades

A. Jocelyn Ritchie

Ami Rokach

Andrea J. Romero

Rosa Rosnati

Sonia L. Rubens

Brenda Russell

Glenda Russell

Pninit Russo-Netzer

Mark Salzer

Rosemary Sarri

Rhoda Scherman

Miriam Schiff

Alayna Schreier

Sarah Schwartz

Robert Schweitzer

Jamie A. Seabrook

Kristie Seelman

Mary V. Seeman

Uma Segal

Gianluca Serafini

Amit Shrira

Natasha Sianko

Derrick Silove

Sondra Smith-Adcock

Cyndy Snyder

Wesley Eugene Sowers

Cesalie Stepney

Deborah Stiles

Bradley Stolbach

Tom Strong

Nasrudin Subhi

Michael J. Sulik

James A. Swartz

Martie Thompson

Ronald W. Thompson

Emily B. H. Treichler

Monica Tsethlikai

Alice P. Villatoro

Deborah Klein Walker

David Wang

Earlise Ward

Martin Wasserberg

Traci Wike

Joshua Mosquera Wilson

Veronica Womack

Svetlana Yampolskaya

Philip Yanos

Ekaterina Yazykova

Irene Nathan Zipper 